\title{
LA COMPETENCIA REGULADA EN EL SISTEMA DE SALUD COLOMBIANO*
}

\author{
Katherine Flórez Pinilla
}

\begin{abstract}
Resumen
Un acercamiento conceptual a los fundamentos microeconómicos del sistema de salud colombiano y especialmente al modelo maneged competition de Alain Enthoven aplicado a Colombia, permite identificar algunas limitantes intrínsecas en el mismo modelo y en la aplicación del mismo, las cuales han determinado el fracaso del sistema de salud actual. Se concluye que en Colombia no ha existido a cabalidad un mercado regulado de la asistencia sanitaria y que la función de regulación, centro del modelo, se desvanece ante las múltiples intervenciones públicas (legislativas, políticas y judiciales) en el sector. Se advierte sobre la necesidad de tener en cuenta dichas problemáticas al momento de diseñar cambios sustanciales -especialmente en estas épocas de reforma- que correspondan a las necesidades de salud de la población colombiana.
\end{abstract}

\section{Palabras clave}

Economía de la salud, regulación, sistema de seguridad social en salud en Colombia, asistencia sanitaria.

\begin{abstract}
A conceptual approach to the microeconomic foundations of the Colombian health system and especially the managed competition model of Alain Enthoven applied to Colombia, to identify some of the inherent limitations in the same model and its implementation, which have determined the failure of the current health system.It concludes that in Colombia there has not been fully regulated market of health care and the role of regulation, core of the model, it vanishes among the many public interventions (legislation, policies and judicial) in the sector. It warns about the need to take into account these issues when designing substantial changes, especially in these times of reform, which correspond to the health needs of our population.
\end{abstract}

\section{Key words}

Health economics, regulation, social security system in health in Colombia, health care.

\footnotetext{
* Producto de la investigación Política y derecho a la salud en Santander 1996-2008 de los grupos de investigación Neoconstitucionalismo, Derecho y "Economía Social". USTA

1 Economista, Docente Investigadora Facultad de Derecho Universidad Santo Tomás. Correo E: katherine09@ mailustabuca.edu.co
} 
¿Tienen en cuenta los reguladores y los diseñadores de políticas públicas la naturaleza económica de los bienes $^{2}$ que pretenden garantizar? ¿Son conscientes de los efectos de sus medidas? El presente artículo busca reflexionar sobre la conceptualización económica que fundamenta el sistema de salud colombiano, habida cuenta que la efectividad de una política pública depende, entre otros factores, del alcance de su dimensión técnica ${ }^{3}$. Para este propósito, se analizará el marco institucional formal que organiza la asistencia sanitaria en Colombia, a la luz de la teoría económica de la salud y la propuesta organizacional de Enthoven.

\section{Los sistemas de salud desde la teoría económica}

Los sistemas de salud se pueden valorar desde el instrumental teórico provisto, tanto por la macroeconomía como por la microeconomía. El primero, es utilizado para evaluar el impacto de la salud en la población de una determinada área, en la producción agregada y en la calidad de vida, entre otras variables que impactan en el crecimiento y desarrollo económico de las sociedades. Por tal motivo, la macroeconomía justifica la oportunidad de la intervención estatal y de destinación de gasto público en el ámbito de la salud humana.

El segundo instrumental se encarga de estudiar la interacción de los agentes y la toma de decisiones de los mismos en un sector, un mercado, empresas, industrias o un conglomerado organizacional. Dado que interesa realizar un análisis organizacional del sistema de salud colombiano, tomaremos como referente la microeconomía, con el fin de estudiar el comportamiento de los agentes y los efectos de las decisiones de los mismos dentro de este sistema salud vigente en Colombia.

\section{Servicios de Salud: ¿Bien privado o bien público?}

La Constitución de la Organización Mundial de la Salud definió la salud como

"un estado de completo bienestar físico, mental y social, y no solamente la ausencia de afecciones o enfermedades (...). El goce máximo de salud que se pueda lograr es uno de los derechos fundamentales de todo ser humano sin distinción de raza, religión, ideología política o condición económica o social."

2 Se utilizará la palabra bienes en un sentido genérico en el cual se incluye bienes tangibles e intangibles, así como servicios.

3 Esto no excluye otro tipo de dimensiones y variables esenciales para el diseño de política, tales como la política y la jurídica, el contexto social e histórico, pero para términos de artículo desarrollaremos este punto en especial, puesto que de acuerdo a Londoño y Frenk $(1997,25)$ "los instrumentos efectivos ponen límites a la discrecionalidad de los tomadores de decisiones".

4 Organización Mundial de la Salud. Constitución de la Organización Mundial de la Salud. 1946. Conferencia Sanitaria Internacional. Restrepo afirma que en la década de los años sesenta se desarrolló un esfuerzo por elaborar un concepto claro de la salud el cual evidenció que "no se puede equiparar la salud y la atención en salud, con la atención médica y los servicios hospitalarios" $(2000,660)$. 
La primera parte excluye el concepto utilitarista de salud que la equipara con la ausencia de enfermedad ${ }^{5}$ y la segunda la concibe como un medio y fin en sí mismo que el Estado debe procurar.

Tal estado deseado de bienestar físico y mental está determinado desde el punto de vista económico ${ }^{6}$, por tres tipos de variables acumulativas tales como "el estilo de vida, las condiciones medioambientales y el consumo de bienes y servicios sanitarios." (Rivera, 2000, 146). Por sus características económicas, se abordará el tercero de los mencionados componentes, a saber, el consumo individual de bienes y servicios sanitarios, o asistencia sanitaria.

Independientemente de las consideraciones políticas, desde la microeconomía la asistencia sanitaria se entiende como un bien privado, en la medida que ostenta un consumo que genera rivalidad y exclusión. Es un bien de consumo rival, porque en la medida que se brinda disminuye su capacidad para brindarse a otros demandantes y es, también, un bien de consumo excluyente, porque el consumo de asistencia sanitaria por unos excluye a otros del consumo del mismo. Un ejemplo de lo anterior es el enfermo que al ocupar una cama hospitalaria excluye a otros enfermos de utilizarla para el mismo fin.

Es decir, el bien asistencia sanitaria es un bien escaso y limitado, excluye y rivaliza el consumo entre personas, por lo cual motiva la competencia para su obtención. Tales bienes, en teoría, deben ser provistos por particulares, que dado su ánimo de lucro también competirán por ofrecer el mejor producto y la mayor cantidad. Esta interacción entre demandantes y oferentes alrededor de un bien, determina automática y espontáneamente el precio y la cantidad óptima de intercambio, a este fenómeno se le conoce como mercado.

En contraste, el estilo de vida y las condiciones medio ambientales constituyen bienes públicos en el sentido económico, su existencia no rivaliza ni excluye a nadie del consumo, su existencia provoca externalidades positivas o negativas. Las primeras, dado un estilo de vida saludable y condiciones medio ambientales favorables que irradia efectos positivos entre las personas alrededor; las segundas cuando existe todo lo contrario, por ejemplo, una persona enferma o la presencia de contaminación en el entorno. Ante tales caracteres de bienes públicos, tradicionalmente la teoría estándar recomienda que deben ser provistos por el Estado, toda vez que de no ser así, habría incentivos al descuido del primero o al derroche del segundo, se

5 En este aspecto el padre del utilitarismo la señala como "Cualidad negativa (...) representada bajo una forma positiva; la salud en nuestro ser físico es la ausencia de toda enfermedad" Bentham, J. (1835, 92).

6 Restrepo afirma que en la década de los años sesenta se desarrolló un esfuerzo por elaborar un concepto claro de la salud el cual evidenció que "no se puede equiparar la salud y la atención en salud, con la atención médica y los servicios hospitalarios" $(2000,660)$. 
evidenciaría la denominada tragedia de los comunes $^{7}$ (Hardin, 1968). No obstante, en la última década se ha demostrado que los bienes públicos excesivamente regulados o intervenidos pueden denotar el efecto contrario, a saber, la tragedia de los anticomunes (Heller, 1998), según la cual la extensa carga de derechos de propiedad sobre un bien incentiva la sub-explotación del mismo (por ejemplo, los bienes patentados).

En un entorno de mercado el consumo de cualquier bien privado normal provee una utilidad marginal decreciente en la medida en que el consumir más del mismo bien, disminuye el grado de satisfacción de cada unidad adicional. La modelización de la utilidad marginal parte de la concepción racionalista del comportamiento humano (Flórez, 2010), según la cual "las personas siempre están en la búsqueda de su interés individual (son egoistas); son racionales, al poder jerarquizar las opciones que tienen de la que genera mayor a la que genera menor beneficio, esto formalmente quiere decir que la persona tiene la capacidad definir y ordenar de manera consistente sus preferencias y de decidir entre alternativas ${ }^{8}$. De lo anterior se deriva que, según la economía ortodoxa, la búsqueda del interés individual, así como la racionalidad instrumental, conlleva a que las personas tengan una conducta tendiente a la maximización de la utilidad.

Con estos supuestos, se deduce que el consumo de bienes y servicios depende del nivel de precios que ostenten, a mayor precio menor cantidad demandada (ley de demanda), no obstante, existen demandas de bienes poco sensibles al precio, la manera de saberlo es calcular la elasticidad precio de la demanda ${ }^{9}$. Así mismo, el efecto causado por el precio de un bien sobre la cantidad demandada de otro, se establece por medio de la elasticidad precio-cruzada, y el efecto de variaciones en la renta sobre la cantidad demandada, se evidencia por la elasticidad renta.

No obstante, tal lógica de los bienes normales no es aplicable para un bien privado como la asistencia sanitaria, dado que la cantidad de asistencia demandada se evidencia principalmente "en la medida en que la salud sufre un deterioro no deseado" (Gimeno, Repullo \& Rubio, 2005, 48), por lo cual, su demanda "no dice

7 En este destacado artículo el ecologista Hardin señala que las personas tienen incentivos a sobreexplotar los bienes de uso común, precisamente por no existir derechos de propiedad definidos sobre los mismos (Hardin, 1968).

8 La microeconomía clásica explica la completitud de las preferencias así: "Si A y B son dos situaciones cualesquiera, el individuo siempre puede especificar exactamente una de las tres posibilidades siguientes: A es preferida a B, B es preferida a A, o A y B son igual de atractivas. Se supone que los individuos no quedan paralizados por la indecisión: comprenden totalmente y siempre pueden decidir sobre la deseabilidad de dos alternativas cualesquiera. El supuesto también excluye la posibilidad de que el individuo pueda afirmar que A es preferida a B y que B es preferida a A" (Nicholson, 2004, 66).

9 Se calcula como el porcentaje en que varía la cantidad demandada de un bien sobre el porcentaje en la variación del precio de dicho bien, si el resultado en términos absolutos es mayor a 1, se dice que la demanda es elástica, si es menor que uno que es inelástica y si es igual a uno que su elasticidad es unitaria. 
nada de lo que la gente está deseando comprar y el valor que a ellos representa el consumo sanitario" (López \& Ortún, 1998, 25). Es decir, existe un componente involuntario que lleva a la persona a un estado de necesidad inminente, esto no excluye la demanda sanitaria por parte de personas sanas, todo lo contrario, en el caso de éstas, el comportamiento del bien tiende a ser elástico, en cambio, la demanda por parte de una persona enferma es inelástica.

De acuerdo a Gimeno et al. $(2005,63)$, la demanda de asistencia sanitaria es una demanda derivada e incluye todo un conjunto de cuidados médicos, tanto preventivos como curativos o terapéuticos, que no se demandan "porque directamente interese, sino con carácter instrumental" con la finalidad de mejorar el estado de salud,

"se demanda salud y, en función de las necesidades de salud, se invierte en ella (...) por medio de comportamientos saludables (inversión neta) o demanda de asistencia sanitaria (inversión bruta)" (Gimeno et al., 2005, 70).

Así mismo, ante la incertidumbre que trae inmersa la posibilidad de tener un estado de enfermedad, se ha recomendado que la mejor forma de procurar la asistencia sanitaria es bajo el mecanismo de aseguramiento ${ }^{10}$, con éste las personas no pagan el valor real de la atención sanitaria demandada sino una prima de seguro ajustada al riesgo de enfermedad, así se realiza una transferencia de recursos entre personas sanas y enfermas (o por frecuencia de utilización). En palabras de Gimeno et al., $(2005,79)$

"el efecto redistributivo disminuye cuanto mayor sea el pago que deba afrontar el asegurado por cada servicio efectivamente utilizado (...) denominado coaseguramiento o copago".

Los efectos asociados al mecanismo de seguro son los mismos que se atribuyen a la presencia de asimetrías de información (Flórez, 2008) en las transacciones. Este fenómeno se evidencia "cuando dos partes de la transacción no comparten el mismo nivel de información” (López \& Ortún, 1998, 33) produce tres problemas básicos: selección adversa, riesgo moral y selección de riesgos.

Según los autores precitados, existen tres tipos de asegurados: los que utilizan los servicios de asistencia médica muy poco, los que generan gastos esporádicos menores y aquellos que necesitan asistencia costosa. Técnicamente, solo éstos últimos pagarán una proporción inferior a la prima del seguro, por lo cual, son los que

10 Sin embargo, no es la única alternativa organizativa para el sector de asistencia sanitaria, "Un seguro de salud es una forma particular de sistema de salud. Existen configuraciones típicas de los sistemas de salud, es decir, formas de organización que tienden a repetirse, donde los diversos actores involucrados asumen roles característicos. Los sistemas de seguros de salud aparecen, cada vez más en la región de las Américas, como soluciones legítimas y eficientes para configurar una respuesta social organizada a las necesidades de salud de la población.” Zerda, Velásquez, Tobar \& Vargas (2001). 
obtienen más ventajas del aseguramiento y más incentivos para adquirir un seguro, este efecto ha sido llamado selección adversa ${ }^{11}$, situación que puede llevar a acabar los mercados, porque las aseguradoras no podrían asignar riesgo de forma eficiente y funcionarían a pérdidas. En el mercado de aseguramiento incidiría, además de la carga de enfermedad de la persona, su actitud hacia el riesgo (adverso, propenso o neutral), como determinante entre la elección aseguramiento vs., compra libre de asistencia sanitaria.

El riesgo moral se evidencia tanto en la falta de cuidados o de prevención individual como en el consumo excesivo de servicios de atención, el aseguramiento "reduce el rendimiento de la inversión neta en salud" por cuanto la persona tiene menos incentivos para prevenir el deterioro de su salud y cuidarse contra contingencias. Además, el costo de la asistencia sanitaria es menor que en la ausencia de seguro, por lo cual tendrá incentivos a consumir más, el precio ya no refleja la información necesaria para racionalizar el consumo, resulta paradójico entonces que "el hecho de asegurar un determinado evento convierte al mismo en más probable" (López \& Ortún, 1998, 34).

El tercer problema asociado a la asimetría en la información se refiere a la selección de riesgos que muestra la tendencia de los aseguradores a seleccionar personas con bajo riesgo de enfermedad que no les ocasione costos excesivos y permita distribuir riesgos de forma favorable financieramente. En este sentido, la demanda (QD) y la oferta (QO) de aseguramiento estarían influenciadas por las siguientes variables (López \& Ortún, 1998):

$Q_{D}=f\left(U_{E}[S] * P[S], R, P\right.$

$$
Q_{o}=f\left(\prod_{E}\right), I, C T
$$

$U_{E}[S]=$ utilidad esperada de tener salud o su ausencia

$P[S]=$ Probabilidad de tener salud o su ausencia $\quad R=$ riqueza disponible

$P=$ valor de la prima de seguro

$\left(\prod_{E}\right)=$ Beneficios esperados

I= indemnización media esperada

$C T=$ costos de transacción

Pero las asimetrías de información no sólo están presentes en el esquema de aseguramiento, en la práctica de la asistencia sanitaria también se evidencian, en la relación médico-paciente, por ejemplo, el médico por sus conocimientos especializados induce al paciente a demandar asistencia y bienes asociados según

11 Con el conocido ejemplo del mercado de los "limones" o de los "cacharros" Akerlof adaptó la ley de comportamiento de Gresham. 
su prescripción, dado que el paciente no posee la información suficiente para valorar la idoneidad, eficacia y oportunidad de la asesoría del médico (Gimeno et. al, 2005, 168), éste último tiene incentivos para actuar de forma oportunista (recomendando medicamentos no efectivos, prolongar la enfermedad y ocultar información, entre otros). A este fenómeno se le conoce como demanda inducida, situación que refleja una decisión que el paciente no tomaría si tuviera toda la información necesaria, por lo cual la demanda no es independiente de la oferta, este fenómeno se conoce como agencia imperfecta (López y Ortún, 1998, 28).

Además de las fallas naturales de la asistencia sanitaria bajo un mercado libre, más las resultantes de introducir el esquema de aseguramiento, Kenneth Arrow (1963) además llamó la atención sobre la presencia de otro tipo de fallo, el referido a la existencia de bienes públicos y externalidades asociadas, tales como el medio ambiente y los cuidados, aquí se origina entonces el componente público del bien salud.

La organización de la asistencia sanitaria tiende naturalmente a estructuras con poder de mercado tales como la competencia imperfecta, entre otras razones, por las barreras a la entrada para proveer asistencia sanitaria, la existencia y el costo de la infraestructura hospitalaria, tecnología, aspectos geográficos y especialidades médicas, entre otras. En síntesis, en un mercado de la salud libre

"la heterogeneidad de los servicios médicos dificulta las comparaciones entre precio y calidad e impide así que se difunda eficazmente la información" (Gimeno et. al, 2005, 169)

y por tanto funcione el libre mercado.

Esta dificultad ha llevado a que el Estado se proponga una política proteccionista sobre la salud, de provisión directa por medio de la expansión de gasto público. Sin embargo, esta medida no está exenta de fallas ya que, en condiciones normales, la provisión gratuita, de bajo costo, incentivará también riesgo moral por mayor consumo de asistencia sanitaria entre los ciudadanos, por lo cual reducirá la eficiencia en la provisión. Así mismo, no escapan tampoco a las fallas intrínsecas del bien "asistencia sanitaria" que se aúnan con las fallas del actuar gubernamental: corrupción y captura.

De acuerdo a la conceptualización anterior, son evidentes las tensiones entre los agentes del mercado de la asistencia sanitaria independientemente de su forma orgánica, éstas surgen de la misma naturaleza y de los intereses contrapuestos de los agentes racionales que interaccionan en el mismo: médicos, pacientes, aseguradoras, prestadoras de servicios, gobierno, tienen cada uno intereses que, al interactuar, generan constantes contradicciones, ante éstas se han diseñado macro-modelos de organización para alinear los incentivos de cada agente e incentivar la cooperación. 


\title{
Fundamentos del sistema de salud colombiano
}

Los fundamentos teóricos del modelo de salud implementado en Colombia a partir de la Ley 100 de 1993, tienen como referente el modelo denominado Managed Competition, conocido como competencia regulada o administrada. Tal ideal de competencia ${ }^{12}$, se origina en los años setenta en los Estados Unidos ${ }^{13}$ en medio de las problemáticas que ostentaban la organización médica de la época, desarrollada bajo un mecanismo de pago por asistencia sanitaria (fee-for-service, Enthoven, 1989, 25), bajo un sistema libre pero no competitivo moldeado por la organización gremial de hospitales y médicos (Blue cross y Blue Shield) y la intervención estatal en la provisión de asistencia (medicare y medicaid). Este modelo se caracterizó por permitir libre elección del médico por parte del paciente, libre elección de la prescripción por el médico, negociación directa de los honorarios entre el médico y el paciente, el control máximo del médico sobre sus ingresos y ejercicio individual de la profesión.

Ante las dificultades que evidenció este esquema libre "sin reglas del juego" (Restrepo, 2000) en la provisión sanitaria, Enthoven (1989) propone el modelo managed competition como una estrategia para que tanto empresarios como consumidores de asistencia sanitaria obtuvieran, dada su racionalidad, el máximo valor en el intercambio, premiando a aquellos planes de salud eficientes que redunden en la equidad del sistema:

\begin{abstract}
(...) Managed competition is a purchasing strategy to obtain maximum value for money for employers and consumers. It uses rules for competition, derived from rational microeconomic principles, to reward with more subscribers and revenue those health plans that do the best job of improving quality, cutting cost, and satisfying patients. The "best job" is in the judgment of both the sponsor, armed with data and expert advice, and informed, cost-conscious consumers. (Enthoven, 1989, 29).
\end{abstract}

El modelo funciona bajo un esquema de aseguramiento de la asistencia sanitaria, en el cual el asegurador, llamado también sponsor, patrocinador o promotor, media entre el usuario y el proveedor de servicios para eliminar las distorsiones presentes en el mecanismo del mercado por medio de reglas del juego.

Bajo este modelo, el Sponsor es el protagonista, tiene el papel de mediador imparcial entre la demanda y la oferta de asistencia, de eliminar las distorsiones propias del mercado de seguros; de valorar la calidad de los planes de provisión de asistencia sanitaria (según información y asesoría de expertos); de definir reglas que incentiven la competencia y no premien planes de salud que seleccionen riesgos, segmenten mercados y provean servicios de mala calidad Enthoven $(1989,30)$. Entre

12 La propuesta de Enthoven fue aplicada en la reforma de la salud de la administración Bill Clinton en 1993

13 Ley de HMO de 1973. Entre otros precursores se encuentran Paul Ellwood, McClure y Walter Scott Fleming.

ENTHOVEN, Alain. The history and principles of managed competition. 
las reglas necesarias para permitir el funcionamiento de un mercado de la salud corregido, Enthoven (1989, 30-35) menciona:

Reglas de equidad: los planes de salud deben aceptar a todas las personas, brindar los precios más bajos posibles (lowestpriced) que aseguren tanto cobertura como calidad; todas las personas pueden reinscribirse durante el período de inscripción anual; la prima se paga por la misma cobertura, independientemente del estado de salud de la persona o pequeño grupo. No hay exclusiones o limitaciones a la cobertura por condiciones preexistentes.

Reglas de selección de planes: los patrocinadores son los encargados de escoger los planes de salud participantes, de acuerdo a criterios de eficiencia y calidad.

Reglas de inscripción: los patrocinadores son los encargados de gestionar la inscripción de planes de salud participantes, así como de los suscriptores. Este agente debe definir los procedimientos de inscripción y dar una oportunidad anual para cambiar de planes. El patrocinador también debe preparar el material informativo sobre los beneficios cubiertos, las características de los planes de salud y la ubicación de sus proveedores, y los controles de calidad efectuados; asimismo debe establecer las condiciones contractuales de pago con la participación de empresarios y particulares, así como servir de intermediario financiero entre los mismos.

Reglas para crear una demanda elástica al precio: para normalizar el comportamiento del producto en el mercado, el patrocinador debe establecer un precio estable (lowestpriced) de la prima de seguro y de reaseguro, así como un producto homogéneo; en este sentido, Enthoven introduce los denominados contratos de cobertura estandarizada, con el fin de disuadir la diferenciación de productos, facilitar la comparación de precios y calidad, y evitar la segmentación del mercado por parte de los productores. La elasticidad de la demanda también se puede formar por medio de información de calidad, libre elección de los planes a nivel individual no por grupos de trabajo.

El patrocinador debe crear incentivos necesarios para que los planes de salud tengan éxito mediante la mejora de la calidad y satisfacción del paciente, no por la selección de riesgos buenos (pacientes sanos) y evasión de los malos (pacientes enfermos). Debe realizar seguimiento de los patrones de inscripción de los usuarios y de los procesos periódicos de afiliación, así como ejercer control de la atención especializada y de calidad; promover la entrada de nuevos actores, manejar subsidios entre sanos y enfermos.

Elsegundo elemento regulado, para permitirel mercado eincentivar la competencia, es el precio del producto que bajo un esquema de aseguramiento corresponde a la prima anual de seguro (Enthoven, 1993, 29), un precio fijo anual pretende que la competencia difiera de la tradicional, pues no se centra en la información de la variabilidad oferta-demanda de asistencia, sino en la calidad y la satisfacción que ofrecen los planes a los usuarios, por lo cual incentivarán a más usuarios a afiliarse. 
Esto implica que los proveedores de salud funcionarán por cantidad demandada, las aseguradoras tendrán incentivos a contratar con aquellos más atractivos para los demandantes, y éstos comprarán el producto o plan de beneficios por mínimo un año y según los resultados los usuarios podrán elegir libremente otra alternativa de prestación del plan. El modelo es compatible con copagos y deducibles seleccionados para los servicios individuales que pueden influir en los pacientes a hacer racional el uso de los recursos (Enthoven, 1993, 30), para reducir el riesgo moral, éstos valores también serían objeto de regulación.

Para Enthoven, la competencia es el mecanismo idóneo para solucionar las falencias del modelo tradicional, propiciar procesos de mejora continua de la productividad y de desarrollo tecnológico, para la solución de enfermedades, por medio de la construcción de un sistema flexible a los cambios y fácil de acceder, dado que los consumidores son agentes idóneos para emitir juicios de calidad por medio de sus elecciones (Enthoven, 1993, 40-41). Con tal modelo, se conseguiría el funcionamiento de un mercado corregido y eficiente, en términos de Enthoven, la eficiencia se convierte en la condición para lograr la equidad en salud, para el autor, éstos no son indicadores excluyentes e incompatibles como tradicionalmente se tratan en el sistema de salud, sino complementarios, según Restrepo, el autor se refiere a una relación articulada y condicionante entre los dos (Restrepo, 1989, 674$675)$.

Al ubicar la presión de la demanda sobre los proveedores para reducir costos, las reformas del mercado de la promoción de la competencia funcionan si todos los que pueden contribuir a la financiación del sistema lo hacen, puesto que -resalta Enthoven- no funcionaría un sistema en que todos estén cubiertos pero sólo unos paguen, incentivaría la presencia de free riders y por tanto la distorsión de modelo. Recomienda el autor que reseñamos, que el pago o las contribuciones de los consumidores podría evidenciarse de diferentes formas, por medio de acuerdos entre empleadores y empleados mediante pagos sobre el salario, subsidios cruzados entre quienes poseen capacidad de pago con quienes no (Enthoven, 1993, 41-42).

\section{Diseño del sistema de salud colombiano}

En Colombia la asistencia sanitaria no ha seguido un patrón organizacional uniforme y armonioso, sino todo lo contrario, ha ostentado formas disfuncionales y fracturadas de organización que se pueden diferenciar en tres grandes fases ${ }^{14}$. La primera fase data del periodo finales del siglo XIX a mediados de siglo XX (1886-1936), y en ella coexistieron, de una parte, la forma privada para aquellos

14 La organización Panamericana de la Salud hace un recorrido histórico sobre las formas de las organizacionales de la asistencia sanitaria en Colombia. Ver HERNÁNDEZ ÁLVAREZ, Mario \& OBREGÓN TORRES, Diana. La Organización Panamericana de la Salud y el Estado Colombiano Cien Años de Historia 1902-2002. 
con capacidad de pago y, de otra, organizaciones de caridad, como la Iglesia, para aquellos sin capacidad de pago, así como la brindada por el sincretismo cultural y prácticas no-científicas por curanderos, chamanes y otros. ${ }^{15}$

La segunda etapa, determinada por la intervención del Estado directamente en la prestación, primero por medio de sistema de seguridad social estatal para los empleados públicos (años cuarenta siglo XX), originario de la idea de Otto von Bismark y, más adelante, con la implementación de un sistema de salud de provisión estatal directa ${ }^{16}$ y centralizada conocido como Sistema Nacional de Salud, creado mediante el Decreto extraordinario 056 del 15 de enero de 1975.

Una tercera fase se enmarca en el fomento de un sistema de cobertura universal de provisión privada bajo regulación estatal por medio de la Ley 100 de 1993, a pesar de las reformas que ha sucedido a esta Ley, tales como la Ley 1122 de 2007 y la Ley 1438 de enero de 2011, no se ha cambiado la naturaleza del modelo guiado por los principios de la competencia regulada de Enthoven.

\section{Nuevo esquema organizacional: el Estado regulador de la Ley 100 de $1993^{17}$}

En Colombia, el proceso de reforma que venía de años atrás en el sector salud ${ }^{18}$ se consolida con la puesta en marcha de la Ley 100 de $1993^{19}$. Su principal novedad son las nuevas funciones del Estado con el sector salud que, en concordancia con los procesos de modernización, (Liberalización, fomento de mercados, apertura y descentralización), pasó de ser prestador directo de servicios de recuperación, a ser garante de la promoción, prevención y recuperación en salud. Estas funciones las desempeña bajo un esquema denominado Seguridad Social, el cual no sólo promoverá el derecho que las personas tienen a la salud (Sistema General de Seguridad Social en Salud [SGSSS]) sino también el derecho que tienen los trabajadores a las pensiones y a la protección contra riesgos profesionales.

15 Es de destacar que desde 1886 el Estado inicia un proceso de intervención en el sector por medio de medidas para la higiene y la salubridad pública, dado el proceso de incursión de la economía colombiana en mercados internacionales (Quevedo, 1989).La Ley 1122 de 2007 reformó la ley 100, no obstante los cambios no se realizaron en el modelo como tal sino es sus elementos organizativos.

16 Londoño y Márquez señalan que el modelo proteccionista de la segunda mitad del siglo pasado "los servicios públicos se prestaban a precios políticos. El Estado aplicaba la solidaridad mediante el subsidio a la oferta, con el fin de hacer llegar los servicios a la población más pobre. En este escenario, las empresas estatales presentaban problemas crónicos de financiación, ineficiencia, burocratización y politización.” MIRANDA LONDOÑO, Alfonso y MÁRQUEZ ESCOBAR, Carlos Pablo. Intervención pública, Regulación administrativa y economía: Elementos para la definición de los objetivos de la regulación. Revista Vniversitas, 2004, 71-116.

17 Cfr. En FLÓREZ PINILLA, Katherine. Liberalización de servicios público de salud: el caso del sector salud en el Área Metropolitana de Bucaramanga. Ponencia [en memorias] presentada en el II Congreso de Gobernabilidad en las Áreas Metropolitanas. Universidad Santo Tomás, Seccional Bucaramanga, Marzo 23-25 de 2011.

18 El proceso está enmarcado por un ambiente de reformas al Estado provocado por el cambio de paradigma

19 La Ley 1122 de 2007 reformó la ley 100, no obstante los cambios no se realizaron en el modelo como tal sino en sus elementos organizativos. 
Para alcanzar el objetivo de garantizar a toda la población colombiana el acceso a los servicios de salud, se propone por ley diseñar un esquema de organización en el cual el Estado regula la prestación, al permitir que tanto firmas privadas ${ }^{20}$ como públicas ${ }^{21}$ puedan funcionar armoniosamente para responder a las necesidades de la población. El esquema propuesto, asumió los lineamientos emergentes de la managed competition, al plantear como fin la cobertura universal en salud, por medio del aseguramiento.

No obstante, la función de patrocinio señalada por Enthoven tomó forma en Colombia de manera fragmentada, unas funciones el Estado por medio de la Comisión de Regulación en salud (CRES) antiguo Consejo Nacional de Seguridad Social en salud y por el Fondo de Solidaridad y Garantía (FOSYGA), otras, un nuevo actor en el sistema: las aseguradoras.

La función reguladora es asumida por el Estado colombiano a través de la CRES, antiguo Consejo de Seguridad Social en Salud (CNSSS) ${ }^{22}$, se refiere a garantizar el funcionamiento de un mercado, con mecanismos regulados como: la función de aseguramiento en manos de particulares, el diseño del producto estandarizado del sistema, denominado Plan Obligatorio de Salud (POS) y la determinación del precio de dicho plan anual, conocido como Unidad de Pago por Capitación (UPC). El diseño incluye las reglas para que los proveedores, prestadores de salud, intermediarios y consumidores funcionen de acuerdo a la lógica del modelo.

La ley 1122 establece, además, la función evaluadora del comportamiento del mercado por medio de medidas de seguimiento periódico por resultados, tanto a intermediarios como a los prestadores públicos del sistema. Por lo cual, dicha figura regulatoria ${ }^{23}$, asume en primera instancia, el papel que Enthoven denomina comité de expertos para definir el producto y el precio del mercado de la salud y se constituye en el protagonista del modelo.

El modelo de prestación de servicios de salud adoptado funciona bajo un esquema de aseguramiento obligatorio a toda la población, pero dadas las diferencias en la capacidad de pago de los habitantes, se establecen tres modalidades de financiamiento. Para afiliar a todas las personas que tienen un contrato de trabajo, a los pensionados y a los trabajadores independientes que se asumen poseen capacidad de pago, se crea el

20 Asumiendo de forma indirecta la responsabilidad de garantizar la atención en salud bajo el esquema de managed competition

21 Esta Ley exigió que las entidades públicas existentes fuesen sometidas a un proceso de reestructuración para adecuarse a un esquema en el cual tuviesen independencia a nivel administrativo, técnico y financiero.

22 En este sentido ver el contenido del artículo 172 de la Ley 100 de 1993, así como la ley 1122 que crea la CRES.

23 En el ordenamiento jurídico colombiano "El regulador es una agencia estatal que actúa para desarrollar el orden económico establecido por la Constitución y la ley. Tanto la función de regulación como la de policía administrativa que se requiere para la efectividad de la primera, están sujetas al principio de legalidad" (Miranda \& Márquez, 2004, 97). 
Régimen Contributivo. Por otro lado, para las personas más pobres y vulnerables del país de las áreas rural y urbana; en situación de desempleo, empleados independientes sin capacidad de pago en el cubrimiento del monto total de la cotización establecida, se les agrupa en el Régimen Subsidiado (Flórez, 2011).

Para aquellas personas con situación económica que no permiten catalogarlos en ninguna de estas modalidades (sin capacidad de pago para contribuir o no lo suficientemente pobre para optar por el subsidiado), la ley dispone un régimen de transición agrupándose en la modalidad de vinculados con acceso a subsidios parciales para obtener beneficios del sistema ${ }^{24}$.

Tal exigencia financiera por subsidios a la demanda exigiría al Estado solvencia, así como capacidad de registro y administración de sistemas de información de los beneficiarios de tales subsidios, para esto se implementó el Sistema de Selección de Beneficiarios (SISBEN) con el propósito de priorizar la demanda.

El segundo mecanismo de la Ley 100 para regular la competencia, es la implementación de un plan de beneficios estándar denominado Plan Obligatorio de Salud (POS) ${ }^{25}$, que incluye atención preventiva, médico-quirúrgica y medicamentos esenciales. No obstante, este plan sería brindado de manera fragmentada de acuerdo a la configuración de los beneficiarios: la forma plena la obtendrían los afiliados al régimen contributivo y de forma parcial los registrados al régimen subsidiado ${ }^{26}$.

Para ofrecer los bienes contenidos en el POS se organiza el sistema bajo dos tipos de agentes: aseguradoras y prestadoras, bajo la denominación de Empresas Promotoras de Salud (EPS) e Instituciones Prestadoras de Servicios (IPS) respectivamente; las primeras patrocinadoras tanto de afiliados contribuyentes (EPS) como subsidiados (EPS), las segundas oferentes de los componentes del POS tanto de naturaleza pública, como privada y solidaria. El artículo 14 de la ley 1122, ratifica la definición del aseguramiento y sus agentes promotores del modelo así:

Artículo 14 [...] entiéndase por aseguramiento en salud, la administración del riesgo financiero, la gestión del riesgo en salud, la articulación de los servicios que garantice el acceso efectivo, la garantía de la calidad en la prestación de los servicios de salud y la representación del afiliado ante el prestador y los demás actores sin perjuicio de la autonomía del usuario.

24 Sobre e ste respecto ver artículo 157, Ley 100 de 1993; artículo 14, literal b., Ley 1122 de 2007. Si bien desde la Ley 100 se establece los denominados vinculados a partir del 2004 se establece una política de subsidios parciales (no inferior al 50\% del valor pleno) para ampliar la cobertura del sistema de salud, éstos subsidios de reglamentan por la Ley 1122 de 2007.

25 Definido en los artículos 156, 162 de Ley 100 de 1993

26 Dada la capacidad financiera del Estado como proveedor directo del pago, se propone entonces que antes del año 2001 recibirían progresivamente los beneficios del POS estándar, iniciando con un $50 \%$ del valor de dicho plan, hasta el año 2001, no obstante a 2011 no se ha logrado la igualación entre planes. 
Las EPS, protagonizan el sistema, tienen entre sus funciones ejercer el patrocinio de oferentes y demandantes, servir de aseguradora de riesgos por enfermedad, distribuirlos entre afiliados, contratar los servicios del POS con IPS, ejercer intermediación financiera, evaluar la calidad de los componentes del POS brindados por las IPS, suministrar información a consumidores para que puedan elegir con libertad y autonomía ${ }^{27}$.

Destaca la norma que independientemente del modo de afiliación, la atención de emergencia se prestará de forma gratuita en todo el territorio; se garantiza al usuario la libre escogencia y traslado entre EPS de acuerdo a ciertas reglas (artículo 25, Ley 1122); la escogencia de las IPS y de los profesionales entre las opciones que cada EPS ofrezca dentro de su red de servicios y la participación de los afiliados en todas las instancias de asociación, representación, veeduría de las entidades rectoras, promotoras y prestadoras y del Sistema de Seguridad Social en Salud. Para las personas que consideren estos beneficios como insuficientes y cuenten con capacidad de pago, se establece en el artículo 169 de la Ley 100, que las EPS “podrán ofrecer planes de salud complementarios al POS, que serán financiados en su totalidad por el afiliado con recursos distintos a las cotizaciones obligatorias".

Para establecer el mecanismo competitivo por calidad, el regulador, en cabeza de la CRES, establece periódicamente el precio del POS por cada afiliado a los regímenes establecidos, denominado Unidad de Pago por Capitación (UPC) ${ }^{28}$, la cual se reglamenta por acto administrativo (artículo 182 de Ley 100, y Ley 1122) de forma periódica, según los cambios en el perfil epidemiológico de la población, los riesgos cubiertos en el plan y los costos de prestación del servicio en condiciones medias de calidad y tecnología.

Para el financiamiento y la administración del régimen contributivo y subsidiado, se establece un fondo estatal: Fondo de Solidaridad y Garantía (FOSYGA). Se dispuso que para el régimen contributivo, la cotización obligatoria (individual o por familia, en concurrencia entre el trabajador y empleador) sería de un valor máximo del $12.5 \%{ }^{29}$ del salario base de cotización (no menor al salario mínimo), dos terceras partes las asume el empleador y una tercera corresponde al trabajador, se dispuso

27 La descripción detallada de la forma como se organiza el Sistema en EPS e IPS se encuentra en el Título II de la Ley 100 de 1993.

28 Artículo 182 Ley 100 de 1993 y Artículo 7, Ley 1122 de 2007.

29 La Ley 1122 en su artículo 10 establece un aumento de $0.5 \%$, originalmente en la Ley 100 se establece el $12 \%$, en contribución del régimen contributivo con respecto a lo estipulado en la Ley 100, el cual se distribuirá de la siguiente forma: al empleador le corresponderá un aporte de $8.5 \%$ y al empleado del $4 \%$, es decir, el empleado sigue contribuyendo igual a la financiación de su seguro establecida en 1993. Parte de tal cotización será ahora igualmente trasladada a la subcuenta de Solidaridad del FOSYGA para contribuir a la financiación del régimen subsidiado pero con un aumento de $0.5 \%$, es decir, apoyará al régimen subsidiado en un monto equivalente a $1,5 \%$. Además, el régimen subsidiado contará con recursos de las entidades territoriales y del FOSYGA, así como de otros recursos de diferente destinación. 
también que un punto de esta cotización sería trasladado al FOSYGA para contribuir a la financiación de los beneficiarios del régimen subsidiado en cumplimiento del principio constitucional de solidaridad.

El FOSYGA, también asume funciones de regulador del mercado al ejercer la intermediación financiera, brindar las reglas de sostenibilidad del sistema, mantener el equilibrio y soportar riesgos por pérdidas financieras en el mercado. El fondo reconoce a las EPS la UPC del POS brindado por afiliado, las EPS recaudan las cotizaciones obligatorias de los afiliados y garantizan los servicios establecidos. De tal cotización descuentan el valor de las UPC fijadas para el POS y trasladan la diferencia al FOSYGA ${ }^{30}$.

La financiación de los subsidios estatales resulta de la cuenta de solidaridad, encargada de administrar los recursos privados del régimen contributivo que se trasladan al régimen subsidiado (subsidio cruzado) y de administrar aportes fiscales destinados directamente para subsidios. La segunda se deriva de los rubros del sistema general de participaciones ${ }^{31}$ de los entes territoriales encargados de seleccionar los beneficiarios $^{32}$; con los recursos obtenidos como producto del monopolio estatal de juegos de azar y los recursos transferidos por ETESA, además según lo dispuesto en la Ley 1438 de 2011 en lo referido al impuesto sobre las armas con destino a la salud, con el fin de aumentar los recaudos ${ }^{33}$.

\section{Fallas del modelo}

\section{Patrocinador imparcial y organización estática}

La pretensión de Enthoven de establecer un mercado regulado evidencia fallas desde sus fundamentos, al considerar de forma estática el mercado de la asistencia sanitaria y no tener en cuenta la importancia de los costos de transacción y de la información para llevar a cabo los intercambios. Un modelo estático interesado sólo en la estructura orgánica, más no en las conductas de cada uno de los agentes, unidas con las características naturales de los bienes que componen el sistema como un todo interrelacionado, se puede catalogar como incompleto e inadecuado.

Aún más, aunque Enthoven parte de las limitantes de la asistencia sanitaria libre, asumiéndolas como "fallos de mercado", pretende eliminar los obstáculos bajo la

30 En caso de ser la suma de las UPC mayor que los ingresos por cotización, el FOSYGA deberá cancelar la diferencia a las EPS que así lo reporten.

31 En principio reglamentado por la Ley 60 de 1993, actualmente por lo dispuesto por la Ley 715 de 2001, Ley 1122 de 2007 y ahora actualizados por Ley 1438 de 2011.

32 Artículo 174 de la Ley 100, según la cual se expone que las direcciones de salud en los entes territoriales organizarán el sistema de subsidios a la población más pobre y vulnerable.

33 Ratificado en el artículo 48 de la Ley 1438 de 2011 según lo estipulado la Ley 100, "Impuesto social a las armas y municiones". 
forma emergente de intervención en la economía, la regulación que permita corregir las fallas y el funcionamiento libre del mercado, no obstante, su modelo no profundiza sobre la conducta de este último modulador del sistema.

La figura del patrocinador como ente imparcial que puede emitir reglas que moldeen la interacción entre oferentes y demandantes hacia la eficiencia, desconoce que el regulador también actúa bajo cierta racionalidad y reglas, lo cual puede llevar a desvío de sus fines, en un contexto institucional como el nacional. Además, la figura del regulador, que para este caso está presente sustancialmente en la CRES, también presenta obstáculos para emitir disposiciones legislativas vinculantes sin que pasen por un proceso político propio del ordenamiento jurídico y de la configuración del poder en Colombia ${ }^{34}$.

En este sentido, Miranda y Márquez (2004) afirman que existen por lo menos cuatro puntos en los cuales los modelos regulatorios emergentes fallan:

“i) el papel otorgado a la administración puede ser más perjudicial que las fallas inherentes del mercado; ii) la incompetencia del regulador para responder a problemas de información en los mercados; iii) "la falacia de la existencia de fallas de mercado" cuando es la forma natural del actuar de los mercados, y iv) "la incapacidad de las agencias de regulación para proteger el interés público cuya guarda les ha sido asignada, resulta ser que dichas autoridades han sido subvertidas por presión, influencia y 'cohecho' para proteger los intereses de aquellos que son sujetos de la regulación” (Miranda \& Márquez, 2004, 98)

Por lo cual, la regulación, muchas veces, pone en desventaja a agentes del mercado e incentiva procesos de corrupción y captura por grupos de presión ${ }^{35}$.

Las anteriores consideraciones se refieren a la posición de regulador, sin embargo, el problema se agrava cuando, caeteris paribus la naturaleza del regulador, éste no tiene en cuenta la presencia de elementos inherentes al intercambio de bienes como los costos de transacción ${ }^{36}$, la racionalidad limitada de los agentes y la presencia de información asimétrica e incompleta entre los mismos, elementos que de muchas formas imposibilitan intercambios simples e incentivan a los agentes a buscar formas alternativas de organización que permitan desarrollar sus actividades de

34 Para ahondar en las razones del fracaso regulatorio ver González $(2007,6)$ quien replica que la CRES tal como fue diseñada no soluciona los problemas del sistema puesto que sus funciones "no tocan aspectos sustantivos, y no ofrecen ningún mecanismo que le permita a la CRES escapar de la inercia institucional existente" resultado de la influencia de los grupos de presión (los mismos agentes del sistema) sobre las decisiones del regulador.

35 Un análisis microeconómico de las denominadas "fallas de gobierno" o de la regulación, se encuentra desde la llamada escuela de la Elección Pública de James Buchanans y Gordon Tullock.

36 Éstos son explicados por Ronald Coase así: "descubrir con quien deseamos transar, informar a la gente que deseamos intercambiar y en qué términos, conducir negociaciones que lleven a un convenio, redactar el contrato, llevar a cabo la inspección necesaria para asegurarnos de que los términos del contrato se observan y demás. Estas transacciones son, a menudo, muy costosas, lo suficiente para evitar muchas transacciones que se llevarían a cabo en un mundo en que el sistema de precios funcionara sin costos". Coase, $(1968,99)$. 
forma óptima. Por lo cual, no se puede pretender diseñar organizaciones y aspirar su estabilidad en el tiempo, si precisamente los agentes cambiarán sus estrategias y actuaciones de acuerdo a procesos de adaptación y evolución. Esta es la lección que trae el análisis neoinstitucionalista de Oliver Williamson para explicar el comportamiento de los mercados y las formas de organización.

Coase explica que la empresa constituye la forma de organización económica alternativa al mercado, que podría lograr los mismos resultados a un costo menor. En este sentido, las empresas no son cuerpos estáticos y equilibrados en el tiempo, tampoco son "una caja negra" como la teoría estándar lo predice. Precisamente, la presencia de costos de transacción e incertidumbre hacen que sean un cuerpo adaptable a los incentivos del entorno, por lo cual este autor advierte que

"una empresa se hace más grande en la medida en que el empresario organiza transacciones adicionales y se hace más pequeña en la medida en que abandona la organización de dichas transacciones (...)"37.

Williamson - citando a Stigler - coincide en que

"la integración vertical será frecuente entre las empresas de industrias jóvenes; a medida que crezca una industria se observará una desintegración; y se producirá una reintegración cuando la industria empiece a decaer" (Williamson, 1991, 33).

Un mundo con información asimétrica y con presencia del oportunismo, lleva a tomar medidas que disminuyan el riesgo que trae consigo el intercambio. Pero éste no es el único problema que afecta el mercado y que, por ende, causa distorsión. Los autores neoinstitucionalistas hablan de la presencia de racionalidad limitada e incertidumbre y de complejidad para entender el medio, como otros de los factores que desvirtúan el modelo neoclásico y que no tiene en cuenta Enthoven. En palabras de Williamson (1991, 38-39):

La racionalidad limitada entraña límites neurofisiológicos, por un lado, y de lenguaje, por el otro. Los límites físicos constituyen límites para valorar y almacenar en la medida de las capacidades para recibir, almacenar, recuperar y procesar información sin errores (...) las limitaciones del lenguaje se refieren a la incapacidad que tienen los individuos para expresar sus conocimientos o sus sentimientos mediante el uso de las palabras, los números o las gráficas de manera que los demás los puedan entender.

Siguiendo al autor precitado, en tal entorno

"la organización interna tiene tres ventajas sobre los métodos de contratación del mercado en circunstancias en las que se unen el oportunismo y las condiciones de números pequeños" $(1991,39)$,

37 COASE, Ronald. La naturaleza de la empresa. P., 562. Op. Cit. 
en primer lugar, evita el comportamiento oportunista de contratantes autónomos: esto debido a los términos de comercio están circunscritos, se evitan posiciones monopolistas; se incentiva la cooperación, se pueden efectuar auditorías de organización interna con más eficacia, así como se pueden arreglar disputas de mejor forma, al desempeñar la organización funciones cuasi-judiciales $(1991,48)$.

El ambiente, además de la información, también influye en la constitución de una organización jerárquica, puesto que las inseparabilidades tecnológicas y de actitudes que reflejan mayor eficiencia en organización planeada que en el mercado (Williamson, 1991).

Tenidos cuenta estos conceptos adicionales que desvirtúan el optimismo de la práctica de mercados perfectos, se puede entender mejor por qué el modelo de Enthoven no ha provisto resultados efectivos en la práctica. La función de aseguramiento a cargo de las EPS no se evidenció tal como predice el modelo, ya que no poseen un rol de intermediario imparcial entre la oferta y la demanda de atención en salud, por el contrario, existe una tendencia a eliminar la función de intermediación, dado que las aseguradoras cuentan con su propia red de prestadoras del servicio de salud o, por lo menos, controlan verticalmente el primer nivel de atención el cual, a su vez, controla los demás niveles de complejidad y tiene contacto directo con el demandante. Esto lleva a que se acentúen los problemas de demanda inducida y demanda derivada propias de la atención sanitaria.

En síntesis, el modelo adaptado a Colombia permite la posibilidad de integración entre EPS e IPS, lo cual permite que en ocasiones las EPS puedan, además de promover la afiliación, prestar directamente servicios tanto del POS, como complementarios (artículos 156, 177, 179 y 181 de Ley 100 de 1993). En este sentido, el artículo 179 de la Ley 100 de 1993, versa:

\begin{abstract}
Para garantizar el POS a sus afiliados, las EPS prestarán directamente o contratarán los servicios de salud con las IPS y los profesionales. Para racionalizar la demanda por servicios, las EPS podrán adoptar modalidades de contratación y pago tales como capitación, protocolos o presupuestos globales fijos, de tal manera que incentiven las actividades de promoción y prevención y el control de costos. Cada Entidad Promotora deberá ofrecer a sus afiliados varias alternativas de IPS, salvo cuando la restricción de oferta lo impida.
\end{abstract}

Este punto ha sido polémico, puesto que los costos de transacción que impone la intermediación en ocasiones impiden la realización de intercambios eficientes, por lo cual, las aseguradoras tendrán incentivos para tener su propia red prestadora de servicios, lo cual puede conllevar a efectos contrarios a la eficiencia social. Las aseguradoras tendrán incentivos para promover sus mismos servicios y no los mejores, según un proceso competitivo entre varias opciones, lo cual restringe el marco de elección del consumidor y puede disminuir la calidad de los servicios. 
Ante este punto la Corte Constitucional se pronunció a favor de tal configuración integrada en Sentencia C 616 de 2001, argumentando que ésta

"No sólo no es contraria a la Constitución (...), sino que la garantía de la libre empresa impide que se obstaculice o prohiba a las EPS la prestación directa del servicio asistencial de salud, por medio de sus IPS, por cuanto ello forma parte de la libertad económica." 38 .

Este hecho ha llevado a la proliferación de la integración vertical, no sólo por las condiciones naturales del mercado sino por incentivo legislativo, como lo señalan Restrepo, Lopera y Rodríguez $(2007,289)$ este grado de integración vertical

"se presenta principalmente en el nivel básico (puerta de entrada) por la ausencia de mercado, ya que no sólo atiende problemas de salud sino que es el eje de coordinación de todo el proceso de atención, o al menos el punto de control que determina el uso de recursos en los niveles superiores".

Además, asegura que las EPS creen IPS para defenderse de la estrechez de los ingresos que reciben por la UPC y de la cartelización de profesionales de la salud e IPS, o por la presencia de activos específicos. Por último, advierte que la integración vertical se puede convertir en

"una oportunidad para capturar rentas por la vía de bajar los costos sin importar la calidad, manteniendo las diferencias entre el gasto médico y la UPC en poder del asegurador" (Restrepo, et al., 2007, 291).

Enfatizan que no sólo el nivel básico es capturado sino que también se ha evidenciado con niveles altos de complejidad, lo cual acentúa el problema al proveer

(...) creación de nueva infraestructura de alta complejidad, lo que tiene un impacto negativo en las finanzas del país porque deja infraestructura ociosa, y tercero, algunas formas de IV, como los contratos por capitación, perjudican los niveles II y III, porque incluyen una transferencia implícita del riesgo al prestador de servicios que no tiene la capacidad financiera ni técnica para asumirlos" (Restrepo, et al., 2007, 291).

La reforma promulgada por la Ley 1122, restringe lo propuesto por la Ley 100, al limitar la integración vertical entre EPS e IPS así:

"Las EPS no podrán contratar, directamente o a través de terceros, con sus propias IPS más del $30 \%$ del valor del gasto en salud. Las EPS podrán distribuir este gasto

38 Declarado exequible por Sentencia C-616-01 de 13 de junio de 2001, Magistrado Ponente Dr. Rodrigo Escobar Gil. 
en las proporciones que consideren pertinentes dentro de los distintos niveles de complejidad de los servicios contemplados en el Plan Obligatorio de Salud" (artículo 15, Ley 1122 de 2007).

Este punto ha sido declarado exequible por la Corte Constitucional mediante Sentencia C-1041 de 2007 al denotar que es acertada la intervención en materia contractual a las EPS, puesto que incentiva la competencia, se pueden obtener resultados de mejora en la calidad, más posibilidades para la libre elección de los usuarios, así como el aprovechamiento de la infraestructura existente y eficiencia en el sistema. Además, la corporación ha reiterado en su jurisprudencia la libertad que tiene el usuario de elegir IPS como expresión del derecho fundamental a la salud consagrado constitucionalmente ${ }^{39}$. Esta disposición ha incidido en el cambio configurativo del sistema, puesto que varias EPS tuvieron que cambiar su estilo de contratación $^{40}$, desintegrarse o simplemente salir del mercado.

\section{Plan Obligatorio de Salud: segmentación y no-correspondencia}

Otro de los fallos regulatorios que se pueden evidenciar al contrastar la fundamentación económica con el modelo desarrollado por la Ley 100, es el diseño de un plan obligatorio de salud como mecanismo para garantizar un producto homogéneo que pudiese ser objeto de competencia, no obstante, el producto no se presta de manera plena a toda la población, con la promesa de progresividad tanto del gasto público como de la capacidad económica del país para disminuir el desempleo hasta el año 2001. A 2011 no se ha conseguido el acceso universal a dicho producto homogéneo. Esta situación ha llevado a notorias inequidades en el acceso a la salud y a masivas vulneraciones a los derechos establecidos constitucionalmente. Así mismo, el POS no es un producto estable, sino que ha variado más de cuarenta veces según datos del Ministerio de Protección Social (2010), por lo cual, existen críticas respecto de su oportunidad e idoneidad para atender las necesidades de salud de la población.

Un indicador de este fracaso se demuestra en la cantidad y contenido de tutelas a través de las cuales se demanda asistencia sanitaria, que evidencia la existencia de barreras para el acceso a la atención en salud efectiva, sea por exclusión explícita paciente por capacidad de pago, porque el servicio solicitado no se encuentra en el POS, o porque éste no lo incluya. La tutela, en este sentido, fue un mecanismo ratificado por Ley 1122 para legitimar la demanda de asistencia sanitaria oportuna de acuerdo a las necesidades sin importar su inclusión o no en el diseño del POS. No

39 Sobre este tema ver Corte Constitucional. Sentencia T-603 de 2010. Magistrado Juan Carlos Henao Pérez.

40 Entre los casos más recientes se encuentra el de "ASMET Salud contra la Asociación de Hospitales de Risaralda, entidad que asocia a las ESE del primer nivel de complejidad de dicho departamento, por la presunta "violación a las normas sobre prácticas comerciales restrictivas de la competencia" traducida en el hecho de que tal asociación intervenía en sus asociadas para que contrataran los servicios de salud con las EPS a una tarifa única y por encima a las tarifas que determina las reglas del mercado, ocasionando abuso en la posición dominante en la región". Noticia disponible en http://www.asmetsalud.org.co/ 
obstante, dada la falta de correspondencia del POS con las necesidades de salud de la población la tutela se ha convertido en un costo de transacción más para acceder al servicio tanto para los usuarios como para el Estado, lo cual es una notoria pérdida de eficiencia. Así, la tutela se ha convertido en un costo de transacción injustificado para el acceso a la salud y medicamentos (Perez, 2007, 816), puesto que tanto para oferentes como demandantes la tutela se ha convertido en un requisito más para obtener servicios de salud.

Tabla 1. Número de tutelas de salud en Colombia 2009

\begin{tabular}{lccccc}
\hline \multirow{2}{*}{ Año } & Total Tutelas & $\begin{array}{c}\text { Tutelas } \\
\text { salud }\end{array}$ & $\begin{array}{c}\% \\
\text { participación }\end{array}$ & Total & Salud \\
\cline { 4 - 6 } $\mathbf{1 9 9 9}$ & 86.313 & 21.301 & $24,7 \%$ & - & - \\
$\mathbf{2 0 0 0}$ & 131.764 & 24.843 & $18,9 \%$ & $52,7 \%$ & $16,6 \%$ \\
$\mathbf{2 0 0 1}$ & 133.272 & 34.319 & $25,8 \%$ & $1,1 \%$ & $38,1 \%$ \\
$\mathbf{2 0 0 2}$ & 143.887 & 42.734 & $29,7 \%$ & $8,0 \%$ & $24,5 \%$ \\
$\mathbf{2 0 0 3}$ & 149.439 & 51.944 & $34,8 \%$ & $3,9 \%$ & $21,6 \%$ \\
$\mathbf{2 0 0 4}$ & 198.125 & 72.033 & $36,4 \%$ & $32,6 \%$ & $38,7 \%$ \\
$\mathbf{2 0 0 5}$ & 224.270 & 81.017 & $36,1 \%$ & $13,2 \%$ & $12,5 \%$ \\
$\mathbf{2 0 0 6}$ & 256.166 & 96.226 & $37,6 \%$ & $14,2 \%$ & $18,8 \%$ \\
$\mathbf{2 0 0 7}$ & 283.637 & 107.238 & $37,8 \%$ & $10,7 \%$ & $11,4 \%$ \\
$\mathbf{2 0 0 8}$ & 344.468 & 142.957 & $41,5 \%$ & $21,4 \%$ & $33,3 \%$ \\
$\mathbf{2 0 0 9}$ & 370.640 & 100.490 & $27,1 \%$ & $7,6 \%$ & $-29,7 \%$ \\
Total & 2.321 .981 & 775.102 & $33,4 \%$ & & \\
\hline
\end{tabular}

Fuente: Tutela y Derecho a la salud. Defensoría del Pueblo, 2009. p. 29.

Por medio de la tutela los jueces solucionan conflictos, muchas veces en contra del diseño institucional emanado de la función regulatoria, por lo cual para algunos, esta intervención resulta perjudicial para el sistema, Pérez $(2007,826)$ argumenta que las tutelas ocasionan inseguridad jurídica, dado que si bien esta figura tiene como objeto

"hacer valer los derechos humanos y los derechos de propiedad (y por ende brindar mayor seguridad jurídica) ha tenido un efecto no esperado: generar una masa inmensa de providencias de la Corte Constitucional" (Pérez, 2007, 876). 
Por medio de la acción de tutela se accede hoy a un sin número de tratamientos que no han sido planteados por medio de la función regulatoria del Estado, cuyos costos deben ser asumidos por el Estado o las EPS, según el caso. Independientemente de la tensión política entre división de poderes que causa la tutela, sus resultados en materia de salud muestran un problema de diseño institucional del producto del sistema.

Tabla 2. Contenido de las tutelas

\begin{tabular}{lcc}
\hline \multirow{2}{*}{$\begin{array}{c}\text { Contenido más frecuente } \\
\text { en las tutelas }\end{array}$} & \multicolumn{2}{c}{ Colombia } \\
\cline { 2 - 3 } Tratamientos & Solicitudes & Part. \% \\
Medicamentos & 8707 & $5,3 \%$ \\
Cirugías & 30025 & $18,4 \%$ \\
Citas médicas & 35288 & $21,7 \%$ \\
Imágenes Diagnósticas & 20340 & $12,5 \%$ \\
Prótesis y ortesis & & $0,0 \%$ \\
Exámenes & 25569 & $15,7 \%$ \\
Procedimientos & 30541 & $18,8 \%$ \\
Otros & 2717 & $1,7 \%$ \\
Total & 9606 & $5,9 \%$ \\
\hline
\end{tabular}

Fuente: Tutela y Derecho a la salud. Defensoría del Pueblo, 2009.

Estos resultados proporcionan señales sobre dificultades técnicas en el diseño del POS y los demás mecanismos pro-mercado del sistema, se pueda plantear la hipótesis según la cual el POS no corresponde al perfil epidemiológico, demografía y entorno poblacional. En este sentido, la falla correspondería al comité de expertos que diseñan el producto homogéneo, es decir, la CRES. Además, si los reguladores tuvieran en cuenta el contenido idóneo del POS para la mayoría de la población, la minoría que no resultara satisfecha en sus necesidades de salud, por medio de la tutela obtendría la atención demandada, así que no existirían incentivos para elegir otro plan de beneficios u otra entidad prestadora, por lo cual se desmorona el espíritu competitivo entre las firmas del sistema, esto es precisamente lo que ocurre en la actualidad. 


\section{Fracturación por capacidad de pago: selección adversa, corrupción y free riders}

El diferenciar a los usuarios por capacidad de pago, otorgar subsidios y cobrar tarifas diferenciadas origina problemas de oportunismo por parte del oferente, tales como la selección adversa y por parte del consumidor, ya que las personas querrán, aunque su situación no lo amerite, obtener subsidios en salud, este caso es constante en el sistema colombiano, el cual se ha evidenciado en el fenómeno de la duplicidad en la afiliación por personas beneficiarias del régimen contributivo, pero con afiliación en el régimen subsidiado para obtener los beneficios del segundo. En este mismo sentido, políticos encargados de los entes territoriales han aprovechado el manejo de los subsidios para ofrecer afiliaciones al régimen a cambio de votos ${ }^{41}$.

\section{Ausencia de capacidad instalada para la asistencia en el entorno rural ${ }^{42}$}

Si las EPS deben patrocinar la oferta de servicios de salud que cumplan el POS y la demanda de servicios de asistencia bajo el mecanismo de aseguramiento y, por tanto, de distribución de riesgos, es totalmente lógico que tiendan a representar grandes grupos poblacionales y que realicen procesos de contratación con la red de prestación existente. Estas dos condiciones se encuentran en los centros urbanos, por lo cual es común encontrar que en la zona rural no existan prestadores privados $\mathrm{y}$, por tanto, mucho menos competencia.

Por ejemplo, en el departamento de Santander los prestadores se encuentran concentrados en la capital, Bucaramanga que

“cuenta con el $42 \%$ de las Instituciones Prestadoras de Salud y en total el Área Metropolitana de Bucaramanga aporta el 55\% de dichas organizaciones. El resto está distribuido en los demás municipios de forma desproporcionada: según información del Ministerio de Protección Social de los 57 municipios de Santander con IPS, 32 cuentan con sólo 1 IPS para brindar asistencia sanitaria a su población, de éstas la mayoría corresponde a Empresas Sociales del Estado o Empresas solidarias" (Flórez, 2011).

La Ley 1438 de 2011 ya menciona esta problemática y recomienda tomar medidas locales de política pública para enfrentar dicha dinámica.

41 Titulares noticiosos como "Familias en Acción y Sisben por votos: Estos dos programas sociales son usados por algunos políticos para favorecer sus campañas.” Revista Semana Jueves 5 Junio 2008 disponible en http://www. semana.com/wf_InfoAr ticulo.aspx?idArt=112421 y “'Trampa o solución de la pobreza?” Revista Dinero, 18 de mayo de 2010, disponible en http://www.dinero.com/edicion-impresa/caratula/trampa-solucion-pobreza_71785. aspx, son el común denominador en la opinión pública colombiana, en la cual se denuncia como en distintas áreas rurales y urbanas son utilizados los subsidios a la demanda como intercambio por votos.

42 Para conocer este fenómeno demostrado en el Departamento de Santander ver Flórez, K. (2011). Liberalización de servicios públicos: el caso del sector salud en el Área Metropolitana de Bucaramanga. Ponencia [en memorias] presentada en el II Congreso de Gobernabilidad en las Áreas Metropolitanas. Universidad Santo Tomás, Seccional Bucaramanga, Marzo 23-25 de 2010. 


\section{Reflexión final}

La reforma del Estado, iniciada desde hace dos décadas, trajo consigo nuevas responsabilidades y funciones para el ente mayor, las responsabilidades emergen de una nueva carta política constitucional y las funciones de los medios propuestos por la misma para alcanzar los fines pactados. En este contexto, se propone un modelo eficientista en el cual el Estado permita a los particulares asumir actividades que en el pasado sostenía, entre éstas la salud fue llamada a un nuevo esquema organizacional.

Bajo este marco, el modelo de Enthoven, o managed competition, tuvo recibo y amplia aplicación en el país, aunque los resultados no fueron los previstos por el mismo. Las fallas en el nuevo esquema organizacional resultan de la confluencia de dos dificultades: falencias conceptuales en el modelo de Enthoven y fallas del Estado en la aplicación del mismo.

Ante la primera falla se hace un llamado al estudio de las características de los bienes, de los agentes, de las instituciones y del entorno en el momento de analizar, estudiar e intervenir en los sectores económicos y, más aún, en sectores con la complejidad que rodea la asistencia sanitaria. La segunda falla, es un elemento olvidado por la teoría económica estándar y, por tal motivo, fuera de contexto en modelos reivindicatorios del mercado. Aquí, la invitación también queda abierta a estudiar el Estado como un agente económico más, dado que está administrado por personas que actúan bajo incentivos económicos, institucionales y emocionales, entre otros, que provea una perspectiva más cercana a la realidad. Una clave para alcanzar este propósito, es incorporar otro tipo de marcos conceptuales a los problemas, tales como las perspectivas de la nueva economía institucional y la escuela austriaca, entre otras, que asumen las limitantes al modelo económico ortodoxo y proveen recomendaciones para su superación.

Así mismo, es importante tener un conocimiento profundo sobre las instituciones formales e informales que enmarcan la actividad económica, así como de las organizaciones que se han creado para actuar en el medio. En la función regulatoria emergente en Colombia, es necesario estudiar la naturaleza y el papel de las Comisiones intersectoriales, en particular la Comisión Regulatoria de Salud, que sin duda tiene un rol protagónico en la forma de organización adoptada en el sector salud.

El modelo de la competencia regulada ha generado prácticas anticompetitivas y no ha permitido el desarrollo de la infraestructura necesaria para responder a las diversas necesidades de salud. Igualmente, es evidente la corrupción presente en el sistema, tanto de lado de la oferta como de la demanda. Se encuentra que no son únicamente los denominados fallos del mercado los obstáculos del sistema, sino precisamente los fallos del Estado en ejercer la función regulatoria las que han determinado rigideces en el sistema e incentivos inadecuados. 


\section{Referencias Bibliográficas}

Arrow, K. (Dic., 1963). Uncertainty and the Welfare Economics of Medical Care. The American Economic Review, 53 (5), 941-973

Bentham, J. (1835). Tratado de las pruebas judiciales. Tomo I. Madrid.

Coase, R. (1968). El problema del costo social. Estudios Públicos [traducción].

Coase, R. (1996). La naturaleza de la empresa. En Oliver E. Williamson, Sidney G. Winter. La naturaleza de la empresa: orígenes, evolución y desarrollo. México: Fondo de Cultura Económica.

Corte Constitucional. Sentencia T-603 de 2010. Magistrado Juan Carlos Henao Pérez. 27 de julio de 2010

Sentencia C-150 de 2003. Magistrado ponente: Manuel José Cepeda Espinosa. 25 de febrero de 2003

.Sentencia T-760 de 2008. Magistrado ponente: Manuel José Cepeda Espinosa. 31 de julio de 2008

Sentencia C-616 de 2001. Magistrado Ponente Dr. Rodrigo Escobar Gil. 13 de junio de 2001.

Defensoría del pueblo. Tutela y Derecho a la salud.2009. disponible en Sitio Web: http://www.defensoria.org.co/red/anexos/publicaciones/ tutelaSalud.pdf

Enthoven, A. (1993). The history and principles of managed competition. Health affairs, 1993, Volumen 13, 24-48.

Flórez, K. (2010). Análisis económico del derecho: antecedentes, teoría y metodología. Ponencia presentada en el II Congreso de Derecho Empresarial y Contractual. Universidad Santo Tomás, Seccional Bucaramanga.

. (2011). Liberalización de servicios público de salud: el caso del sector salud en el Área Metropolitana de Bucaramanga. Ponencia [en memorias] presentada en el II Congreso de Gobernabilidad en las Áreas Metropolitanas. Universidad Santo Tomás, Seccional Bucaramanga, Marzo 23-25 de 2010

. (2008). Prestación de servicios de salud en Colombia: dilema entre el estado y mercado. IUSTITIA, 6, 163-182.

Gimeno, J. A., Repullo, J. R., Rubio, S. (2005). Economía de la salud: fundamentos. Manuales de dirección médica y gestión clínica. 2005. España: Editorial Díaz de Santos.

González, J. I. (2007). La regulación en salud en ausencia del velo de la ignorancia. Ponencia presentada en Jornada Inaugural de ACOES. Junio. 
Hardin, G. (1968). La tragedia de los comunes. Este artículo fue publicado en Science, 162, 1243-1248.

Heller, M. (1998). The Tragedy of the Anticommons: Property in the Transition from Marx to Markets. Harvard Law Review, 621-688.

Hernández, M. \& Obregon, D. (2002). La Organización Panamericana de la Salud y el Estado colombiano cien años de historia 1902-2002. Organización Panamericana de la Salud.

Ley 100 de 1993. Por la cual se crea el sistema de seguridad social integral y se dictan otras disposiciones. Diario Oficial No. 41.148 de 23 de diciembre de 1993

Ley 1122 de 2007. Por la cual se hacen algunas modificaciones en el Sistema General de Seguridad Social en Salud y se dictan otras disposiciones. Diario Oficial No. 46.506 de 9 de enero de 2007

Ley 1438 de 2011. Por medio de la cual se reforma el sistema general de seguridad social en salud y se dictan otras disposiciones. 19 enero de 2011.

Londoño, J. L., \& Frenk, J. (1997). Pluralismo estructurado: hacia un modelo innovador para la reforma de los sistemas de salud en América Latina. Banco Interamericano de Desarrollo. Documento de trabajo 353.

López, G. \& Ortún, V. (1998). Economía y salud: fundamentos y políticas. Madrid: Ediciones Encuentro.

Ministerio de protección social (2010). Actualizaciones y Aclaraciones al POS-S y POS-C 1994-2000. Sitio Web Plan Obligatorio de Salud, disponible en: http:// www.pos.gov.co/Documents/Actualizaciones\% 20y\%20aclaraciones $\% 20 \mathrm{al} \% 20$ POS-S\%20y\%20POS-C\%201994-2010. pdf

Miranda, A. y Márquez, C. P. (2004). Intervención pública, Regulación administrativa y economía: Elementos para la definición de los objetivos de la regulación. Revista Vniversitas, 71-116.

Nicholson, W. (2004). Teoría Microeconómica: principios básicos y ampliaciones. [8 8 ed.]. Thomson.

Organización Mundial de la Salud (1946). Constitución de la Organización Mundial de la Salud. 1946. Conferencia Sanitaria Internacional. Disponible en sitio web.

Pérez, M. (2007). Economía y fallos constitucionales. La experiencia de la corte colombiana durante la vigencia de la Carta Política de 1991. En Teoría Constitucional y políticas públicas. Bases críticas para una discusión. Manuel José Cepeda, Eduardo Montealegre, Alexei Julio. Universidad Externado de Colombia. 
Restrepo, M. (2000). Ensayos sobre el financiamiento de la seguridad social en salud. Los casos de Estados Unidos, Canadá, Argentina, Chile, Colombia. Volumen II. Daniel Ttelman y Andras Uthoff [compiladores]. Comisión Económica para América Latina y el Caribe. Santiago de Chile: Fondo de Cultura Económica.

Restrepo, J. H., Lopera, J. F., Rodríguez, S. M. (Jul.-Dic., 2007). La integración vertical en el sistema de salud colombiano. Revista Economía Institucional, 09 (17), 2007, 279-308.

Rivera, B. (2000). Estado de salud, gasto sanitario y sus implicaciones en la productividad: teoría y evidencia. Universidad Pompeu Fabra. Departamento de Economía y Empresa. [Tesis doctoral]. Barcelona. Recuperado de http://www. tesisenred.net/TESIS_UPF/AVAILABLE/ TDX-0715110-094711//tbrc2de2.pdf el día 10 de julio de 2010 .

Williamson, O. (1991). Mercados y jerarquías: su análisis y sus implicaciones antitrust. México: Fondo de Cultura Económica.

Quevedo, (1989). La salud en Colombia: análisis socio-histórico. Revista salud Colombia [Informe especial].

Zerda, A., Velásquez, G, Tobar, F., \& Vargas, J. E. (2001). Sistemas de seguros de salud y acceso a medicamentos. Estudios de casos Argentina, Colombia, Costa Rica, Chile, Estados Unidos de América y Guatemala. Recuperado de http:// apps.who.int/medicinedocs/en/d/ Jh2958s/4.1.html el día 9 de agosto de 2010. 